\title{
Ueber die Ergänzungssätze zu den allgemeinen Reciprocitätsgesetzen.
}

\author{
(Von Herrn E. E. Kummer.)
}

In der Abhandlung Bd. 44, pag. 93 dieses Journals, welche denselben Titel führt, habe ich gezeigt, dafs die auf irgend eine ideale oder wirkliche Primzahl $f(\alpha)$, in der Theorie der aus $\lambda^{\text {ten }}$ Wurzeln der Einheit gebildeten complexen Zahlen, sich beziehenden Indices der Zahlen $1-\alpha, \lambda$ und der complexen Einheiten durch die Zahlen der Kreistheilung in einfacher Weise bestimmt werden. Da aber diese Bestimmung nur auf die idealen Primfactoren der nichtcomplexen Primzahlen $p$, von der Form $p=m \lambda+1$, Anwendung findet, so habe ich durch eine Verallgemeinerung der Theorie der Kreistheilung die entsprechenden Resultate auch für alle diejenigen idealen Primzahlen $f(\alpha)$ gewonnen, welche Primfactoren nichtcomplexer Primzablen von anderen linearen Formen sind. Ferner hahe ich die Indices eines bestimmten unabhängigen Systems von Einheiten, durch welches alle Einheiten sich ausdrücken lassen, auch so dargestellt, dafs sie nicht mehr durch die Zahlen der Kreistheilung, sondern durch die complexe Primzahl $f(\alpha)$ selbst bestimmt werden, auf welche der Index sich bezieht. Seitdem habe ich bei Gelegenheit eines Beweises der allgemeinen Reciprocitätsgesetze für $\lambda^{\text {te }}$ Potenzreste unter je zwei complexen Primzahlen, den ich im Februar 1858 in der Königlichen Akademie der Wissenschaften vorgetragen habe, auch für die Ergänzungssätze zu denselben einige neue, beachtenswerthe Ausdrücke gefunden, welche ich hier kurz entwickeln will.

\section{1.}

Setzt man in der bekannten Lagrangeschen Resolvente der Kreistheilung

$$
\boldsymbol{F}(\alpha, x)=\boldsymbol{x}+\alpha \boldsymbol{x}^{y}+\alpha^{2} x^{g^{2}}+\cdots+\alpha^{p-2} x^{g^{p-2}},
$$

in welcher $\alpha$ eine primitive Wurzel der Gleichung $\alpha^{\alpha}=1, x$ eine primitive Wurzel der Gleichung $x^{p}=1, \lambda$ eine ungrade Primzahl, $p$ eine Primzahl der Form $p=m \hat{\lambda}+1$ und $g$ eine primitive Wurzel von $p$ ist, statt der 
Wurzel $\alpha$ die variable Exponentialgröfse $e^{v}$ und bildet den $\lambda^{\text {ten }}$ Differentialquotienten des Logarithmus von $\boldsymbol{F}\left(\boldsymbol{e}^{v}, \boldsymbol{x}\right)$, so erhält man, nach Weglassung der mit dem Factor $\lambda$ behafteten Glieder, die Congruenz:

$$
\text { (1.) } \quad \frac{d^{\lambda} l \boldsymbol{F}\left(c^{v}, x\right)}{d v^{\lambda}} \equiv \frac{d^{\lambda} \boldsymbol{F}\left(e^{v}, x\right)}{\boldsymbol{F}\left(\boldsymbol{e}^{\nu}, x\right) d v^{\lambda}}-\left(\frac{d \boldsymbol{F}\left(e^{v}, x\right)}{\boldsymbol{F}\left(\boldsymbol{e}^{v}, x\right) d v}\right)^{\lambda}, \quad(\bmod . \lambda) \text {. }
$$

Man hat nämlich nach einer bekannten Formel der Differentialrechnung, wenn $\boldsymbol{U}$ eine Function von $u$, und $\boldsymbol{u}$ eine Function von $v$ ist:

$$
\text { (2.) } \frac{d^{\lambda} U}{d v^{\lambda}}=C_{1} \frac{d U}{d u}+C_{2} \frac{d^{2} U}{d u^{2}}+C_{3} \frac{d^{3} U}{d u^{3}}+\cdots+C_{\lambda} \frac{d^{\lambda} U}{d u^{\lambda}} \text {, }
$$

wo $C_{1}, C_{2}, C_{3}, \ldots C_{\lambda}$ aus den Differentialquotienten des $u$ nach $v$ zusammengesetzt sind, und namentlich

$$
C_{1}=\frac{d^{\lambda} u}{d v^{\lambda}}, \quad C_{\lambda}=\left(\frac{d u}{d v}\right)^{\lambda}
$$

ist, und wo, wenn $\lambda$ Primzahl ist, die Gröfsen $C_{2}, C_{3}, \ldots C_{\lambda-1}$ alle den Zahlenfactor $\lambda$ enthalten. Aus dieser Gleichung folgt die obige Congruenz unmittelbar, wenn $u=\boldsymbol{F}\left(\boldsymbol{e}^{v}, \boldsymbol{x}\right), \boldsymbol{U}=\boldsymbol{l} \boldsymbol{F}\left(\boldsymbol{e}^{v}, \boldsymbol{x}\right)=\boldsymbol{l}(\boldsymbol{u})$ genommen wird, und wenn man beachtet, dafs $1.2 .3 \ldots(\lambda-1) \equiv-1,(\bmod . \lambda)$ ist.

Setzt man nun $v=0$, so wird:

$$
\begin{gathered}
\boldsymbol{F}(1, x)=x+x^{g}+x^{g^{2}}+\cdots \cdots \cdots \cdots+x^{g^{p-2}}=-1, \\
\frac{d_{0} \boldsymbol{F}\left(e^{v}, x\right)}{d v}=x^{g}+2 x^{g^{2}}+3 x^{g^{3}}+\cdots+(\boldsymbol{p}-2) x^{g^{p-2}}=\sum_{0}^{p-2} h x^{g^{h}}, \\
\frac{d_{0}^{\lambda} \boldsymbol{F}\left(\boldsymbol{e}^{v}, x\right)}{d v^{\lambda}}=x^{g}+2^{\lambda} x^{g^{2}}+3^{\lambda} x^{g^{3}}+\cdots+(\boldsymbol{p}-2)^{\lambda} x^{g^{p-2}}=\sum_{0}^{p-2} h^{\lambda} x^{g^{h}},
\end{gathered}
$$

also vermöge der Congruenz $h^{\lambda} \equiv h,(\bmod . \lambda)$,

$$
\frac{d_{0}^{\lambda} F\left(e^{v}, x\right)}{d v^{\lambda}} \equiv \frac{d_{0} F\left(e^{v}, x\right)}{d v} \equiv \sum_{0}^{p-2} h x^{g^{h}}, \quad(\bmod . \lambda) ;
$$

die Congruenz (1.) giebt daher:

$$
\text { (3.) } \quad \frac{d_{0}^{\lambda} l F\left(e^{v}, x\right)}{d v^{\lambda}} \equiv-\sum_{0}^{p-2} h x^{g^{h}}+\left(\sum_{0}^{p-2} h x^{g^{h}}\right)^{\lambda}, \quad(\bmod . \lambda) \text {. }
$$

Die $\lambda^{\text {te }}$ Potenz eines Polynoms ist aber, wenn $\lambda$ Primzahl ist, der Summe der $\lambda^{\text {ten }}$ Potenzen der einzelnen Glieder desselben congruent; man hat daher

$$
\left(\sum_{0}^{p-2} h x^{g^{h}}\right)^{\lambda} \equiv \sum_{0}^{p-2} h^{\lambda} x^{\lambda g^{h}}, \quad(\bmod . \lambda)
$$

oder wenn $\lambda$, als Potenz der primitiven Wurzel dargestellt,

$$
\text { (4.) } \lambda \equiv g^{i}, \quad(\bmod . p)
$$


giebt, und wenn $h$ statt $h^{\lambda}$ gesetzt wird,

$$
\left(\sum_{0}^{p-2} h x^{g^{h}}\right)^{\lambda} \equiv \sum_{0}^{p-2} h x^{g^{h+i}}, \quad(\bmod . \lambda) \text {. }
$$

Verwandelt man $h$ in $h-i$, so wird

$$
\sum_{0}^{p-2} h x^{g^{h+i}}=\sum_{0}^{p-2} h x^{g^{h}}-i \sum_{0}^{p-2} x^{g^{h}}=\sum_{0}^{p-2} h x^{g^{h}}+i,
$$

die Congruenz (3.) giebt demnach

$$
\frac{d_{0}^{\lambda} l F\left(e^{v}, x\right)}{d v^{\lambda}} \equiv i, \quad(\bmod . \lambda) .
$$

Es sei nun $f(\alpha)$ einer der $\lambda-1$ conjugirten idealen Primfactoren des $p$, und zwar derjenige, welcher zur Congruenzwurzel $\alpha=g^{\frac{p-1}{\lambda}}$ gehört, es bezeichne ferner Ind. den Index, welcher sich auf die Primzahl $f(\alpha)$ bezieht, so dafs

$$
\left(\frac{\lambda}{f(\alpha)}\right)=\alpha^{\operatorname{lnd} . \lambda} \equiv \lambda^{\frac{N f(\alpha)-1}{\lambda}}, \quad(\bmod . f(\alpha))
$$

ist, so hat man, weil $N f(\alpha)=p$ und $\alpha \equiv g^{\frac{p-1}{\lambda}},(\bmod . f(\alpha))$ ist,

$$
\lambda^{\frac{p-1}{\lambda}} \equiv g^{\frac{p-1}{\lambda} \operatorname{lnd} . \lambda}
$$

- für den Modul $f(\alpha)$, und darum auch für den Modul $\boldsymbol{p}$. Andererseits giebt die Congruenz (4.), durch welche die Zahl $i$ bestimmt ist,

$$
\lambda^{\frac{p-1}{\lambda}} \equiv g^{\frac{p-1}{\lambda} i}, \quad(\bmod . p)
$$

und aus der Vergleichung dieser beiden Congruenzen folgt:

$$
i \equiv \text { Ind. } \lambda, \quad(\bmod . \lambda) \text {. }
$$

Die Congruenz (5.) giebt daher folgenden merkwürdigen Ausdruck des Index von $\lambda$ in Beziehung auf $f(\alpha)$ :

$$
\text { (6.) Ind. } \lambda \equiv \frac{d_{0}^{\lambda} l F\left(e^{v}, x\right)}{d v^{\lambda}}, \quad(\bmod . \lambda) \text {. }
$$

\section{2.}

Aus dem gefundenen, dem Gebiete der Kreistheilung angehörenden Ausdrucke des Index von $\lambda$ erhält man einen entsprechenden durch die ideale Primzahl $f(\alpha)$, auf welche der Index sich bezieht, unmittelbar bestimmten Ausdruck, vermittelst der Zerlegung der $\lambda^{\text {ten }}$ Potenz von $F(\alpha, x)$ in ihre 
idealen Primfactoren, nach der Formel

(7.) $\quad F(\alpha, x)^{\lambda}= \pm \alpha^{s} f\left(\alpha^{-1}\right)^{m_{1}} f\left(\alpha^{-2}\right)^{m_{2}} f\left(\alpha^{-3}\right)^{m_{3}} \ldots f\left(\alpha^{-(\lambda-1)}\right)^{m_{\lambda-1}}$, in welcher die Exponenten $m_{h}$ als die kleinsten positiven Wurzeln der Congruenzen von der Form

$$
h m_{h} \equiv 1, \quad(\bmod . \lambda)
$$

bestimmt sind und $f(\alpha)$ derjenige ideale Primfactor des $p$ ist, welcher der: Congruenzwurzel $\alpha=g^{\frac{p-1}{\lambda}}$ angehört. Um die idealen Factoren dieses Products zu wirklichen zu machen, wird auf beiden Seiten zur $\boldsymbol{H}^{\text {ten }}$ Potenz erhoben, wo $\boldsymbol{H}$ der Exponent derjenigen Potenz sein soll, für welche $f(\alpha)^{H}=\varphi(\alpha)$ eine wirkliche complexe Zahl ist. Es soll auch angenommen werden, dafs $\boldsymbol{H}$ nicht durch $\lambda$ theilbar ist, welche Bedingung bekaṇntlich immer erfüllt ist, wenn $\lambda$ nicht ein Factor des Zählers einer der ersten $\frac{\lambda-3}{2}$ Bernoullischen Zahlen ist. Verwandelt man alsdann die Wurzel $\alpha$ in die variable Exponentialgröfse $e^{v}$, so erhält man aus der Gleichung (7.) in bekannter Weise eine für jeden beliebigen Werth der Variabeln $e^{v}$ geltende Gleichung von der Form

in welcher

$$
\boldsymbol{F}\left(\boldsymbol{e}^{v}, \boldsymbol{x}\right)^{H \lambda}= \pm \boldsymbol{e}^{H s v} \boldsymbol{\Pi}_{\boldsymbol{1}}^{\lambda-1} \varphi\left(\boldsymbol{e}^{-h v}\right)^{m} h+\boldsymbol{V} \cdot \boldsymbol{W}
$$

$$
\boldsymbol{V}=1+\boldsymbol{e}^{v}+\boldsymbol{e}^{2 v}+\cdots+\boldsymbol{e}^{(\lambda-1) v}
$$

und $\boldsymbol{W}$ eine ganze rationale Function von $e^{v}$ und von der Wurzel $x$ ist. Nimmt man auf beiden Seiten die Logarithmen, so erhält man

wo

$$
\boldsymbol{H} \lambda l \boldsymbol{l}\left(\boldsymbol{e}^{v}, x\right)=\boldsymbol{l}( \pm 1)+\boldsymbol{H} s v+\sum_{1}^{\lambda-1} m_{h} l \varphi\left(e^{-h v}\right)+l\left(1+V W_{1}\right)
$$

$$
W_{1}=\frac{W}{ \pm e^{H s v} \Pi_{h} \varphi\left(e^{-h v}\right)^{m_{h}}},
$$

und wenn der $\lambda^{\text {te }}$ Differentialquotient nach $v$ genommen wird:

$$
\text { (9.) } \quad \boldsymbol{H} \lambda \frac{d^{\lambda} l \boldsymbol{F}\left(e^{v}, x\right)}{d v^{\lambda}}=\sum_{1}^{\lambda-1} m_{h} \frac{d^{\lambda} l \varphi\left(e^{-h v}\right)}{d v^{\lambda}}+\frac{d^{\lambda} l\left(1+V W_{1}\right)}{d v^{\lambda}} \text {. }
$$

Nimmt man nun $v=0$ und macht aus dieser Gleichung eine Congruenz nach dem Modul $\lambda^{2}$, so hat man zunächst

$$
\text { (10.) } \quad \frac{d_{0}^{\lambda} l\left(1+V W_{1}\right)}{d v^{\lambda}} \equiv 0, \quad\left(\bmod . \lambda^{2}\right),
$$


für jeden beliebigen $W$ erth des $W_{1}$, welcher ganz oder gebrochen sein kann, wenn nur, wie es hier der Fall ist, der Nenner desselben für $v=0$ nicht den Factor $\lambda$ enthält. Entwickelt man nämlich diesen Differentialquotienten des Logarithmus nach der allgemeinen Formel (2.), so erhält man

$$
\frac{d^{\lambda} l\left(1+V W_{1}\right)}{d v^{\lambda}}=\frac{\frac{d^{\lambda}\left(V W_{1}\right)}{d v^{\lambda}}}{1+V W_{1}}-\frac{\lambda \frac{d^{\lambda-1}\left(V W_{1}\right)}{d v^{\lambda-1}} \frac{d\left(V W_{1}\right)}{d v}}{\left(1+V W_{1}\right)^{2}}+\cdots
$$

wo alle folgenden Glieder Producte von mindestens zwei der ersten $\lambda-2$ Differentialquotienten von $V W_{1}$ im Zähler enthalten. Diese $\lambda-2$ ersten Differentialquotienten sind aber für den Werth $v=0$ alle durch $\lambda$ theilbar, weil $V$ mit seinen ersten $\lambda-2$ Differentialquotienten für $v=0$ durch $\lambda$ theilbar ist. Hieraus folgt, dafs für $v=0$ jedes der auf das erste folgenden Glieder dieser Entwicklung den Factor $\lambda$ mindestens zweimal enthält, dafs also

$$
\frac{d_{0}^{\lambda} l\left(1+V W_{1}\right)}{d v^{\lambda}} \equiv \frac{d_{0}^{\lambda}\left(V W_{1}\right)}{d v^{\lambda}} \cdot \frac{1}{1+\lambda W_{1}}, \quad\left(\bmod . \lambda^{2}\right) \text {. }
$$

Ferner hat man nach einer bekannten Formel für die Differentiation eines Products zweier Factoren:

$$
\frac{d^{\lambda}\left(\boldsymbol{V} \boldsymbol{W}_{1}\right)}{d v^{\lambda}}=\frac{d^{\lambda} \boldsymbol{V}}{d v^{\lambda}} \boldsymbol{W}_{1}+\lambda \frac{d^{\lambda-1} \boldsymbol{V}}{d v^{\lambda-1}} \frac{d \boldsymbol{W}_{1}}{d v}+\frac{\lambda(\lambda-1)}{1.2} \frac{d^{\lambda-2} \boldsymbol{V}}{d v^{\lambda}-2} \frac{d^{2} \boldsymbol{W}_{1}}{d v^{2}}+\cdots+\boldsymbol{V} \frac{d^{\lambda} \boldsymbol{W}_{1}}{d v^{\lambda}}
$$

und weil die Differentialquotienten des $V$ bis zum $\lambda-2^{\text {ten }}$ einschliefslich für $v=\mathbf{0}$ alle den Factor $\lambda$ enthalten, und die Binomialcoefficienten der $\lambda^{\text {ten }}$ Potenz denselben ebenfalls enthalten, so hat man

$$
\frac{d_{0}^{\lambda}\left(V W_{1}\right)}{d v^{\lambda}} \equiv \frac{d_{0}^{\lambda} V}{d v^{\lambda}} W_{1}+\lambda \frac{d_{0}^{\lambda-1} V}{d v^{\lambda-1}} \frac{d_{0} W_{1}}{d v}+\lambda \frac{d_{0}^{\lambda} W_{1}}{d v^{\lambda}}, \quad\left(\bmod . \lambda^{2}\right) .
$$

Es ist aber

$$
\begin{aligned}
& \frac{d_{0}^{\lambda} V}{d v^{\lambda}}=1^{\lambda}+2^{\lambda}+3^{2}+\cdots+(\lambda-1)^{\lambda} \equiv 0, \quad\left(\bmod . \lambda^{2}\right), \\
& \frac{d_{0}^{\lambda-1} V}{d v^{\lambda-1}}=1^{\lambda-1}+2^{\lambda-1}+3^{\lambda-1}+\cdots+(\lambda-1)^{\lambda-1} \equiv-1, \quad(\bmod . \lambda) \\
& \frac{d_{0}^{\lambda} W_{1}}{d v^{\lambda}} \equiv \frac{d_{0} W_{1}}{d v}, \quad(\bmod . \lambda)
\end{aligned}
$$

folglich

und darum auch

$$
\frac{d_{0}^{i}\left(V W_{1}\right)}{d v^{2}} \equiv 0, \quad\left(\bmod . \lambda^{2}\right)
$$

$$
\frac{d_{0}^{\lambda} l\left(1+V W_{1}\right)}{d v^{\lambda}} \equiv 0, \quad\left(\bmod . \lambda^{2}\right)
$$

wie behauptet worden. 
Nimmt man nun die ideale Zahl $f(\alpha)$, als $\boldsymbol{H}^{\text {te }}$ Wurzel aus der wirklichen Zahl $\varphi(\alpha)=f(\alpha)^{H}$ dargestellt, in der. Form $f(\alpha)=\sqrt[H]{\varphi(\alpha)}$, so kann man einfach

$$
\frac{1}{H} \frac{d_{0}^{\lambda} l \varphi\left(e^{-h v}\right)}{d v^{\lambda}}=\frac{d_{0}^{\lambda} l f\left(e^{-h v}\right)}{d v^{\lambda}}
$$

bezeichnen, und weil

$$
\frac{d_{0}^{\lambda} l f\left(e^{-h v}\right)}{d v^{\lambda}}=-h^{\lambda} \frac{d_{0}^{\lambda} l f\left(e^{v}\right)}{d v^{\lambda}}
$$

ist, so giebt die Gleichung (9.) folgende Congruenz:

$$
\frac{\lambda d_{0}^{\lambda} l \boldsymbol{F}\left(\boldsymbol{e}^{v}, x\right)}{d v^{\lambda}} \equiv-\frac{d_{0}^{\lambda} l f\left(e^{v}\right)}{d v^{\lambda}} \sum_{1}^{\lambda-1} h^{\lambda} m_{h},\left(\bmod . \lambda^{2}\right)
$$

also vermöge der Formel (6.)

$$
\lambda \text { Ind. } \lambda \equiv-\frac{d_{0}^{\lambda} l f\left(e^{v}\right)}{d v^{\lambda}} \sum_{l}^{\lambda-1} h^{\lambda} m_{h}, \quad\left(\bmod . \lambda^{2}\right)
$$

Weil $h m_{h} \equiv 1,(\bmod . \lambda)$, so hat man

$$
\sum_{1}^{\lambda-1} h^{\lambda} m_{h} \equiv \sum_{1}^{\lambda-1} h^{\lambda-1} \equiv-1, \quad(\bmod . \lambda)
$$

hieraus schliefst man zunächst, dafs $\frac{d_{0}^{\lambda} l f\left(e^{v}\right)}{d v^{\lambda}}$ durch $\lambda$ theilbar sein mufs, welches auch anderweitig leicht $\mathrm{zu}$ beweisen ist; dividirt man also durch $\lambda$, so hat man folgenden neuen Ausdruck des Index von $\lambda$ :

$$
\text { (11.) Ind. } \lambda \equiv \frac{1}{\lambda} \frac{d_{0}^{\lambda} l f\left(e^{v}\right)}{d v^{\lambda}}, \quad(\bmod . \lambda) \text {. }
$$

Obgleich die Methode der Herleitung dieses Ausdrucks voraussetzt, dafs $f(\alpha)$ ein complexer Primfactor einer nichtcomplexen Primzahl $p$ der linearen Form $m \lambda+1$ ist, so gilt derselbe dennoch ebenfalls für alle anderen idealen Primzahlen, weil für alle Primzahlen $f(\alpha)$, welche complexe Primfactoren nichtcomplexer Primzahlen anderer linearer Formen sind, stets zugleich

$$
\text { (12.) Ind. } \lambda \equiv 0 \text { und } \frac{1}{\lambda} \frac{d_{0}^{\lambda} l f\left(e^{v}\right)}{d v^{\lambda}} \equiv 0, \quad(\bmod . \lambda) \text {, }
$$

statt hat. Es folgt diefs daraus, dafs die conjugirten complexen Primfactoren einer jeden nichtcomplexen Primzahl $q$, welche nicht von der Form $m i+1$ ist, zum Theil einander gleich sind, dafs also für dieselben eine Gleichung von der Form

$$
f(\alpha)=f\left(\alpha^{r}\right)
$$


statt hat, in welcher $r$ nicht congruent Eins nach dem Modul $\lambda$ ist. Vermöge dieser Gleichung hat man

$$
\frac{1}{\lambda} \frac{d_{0}^{\lambda} l f\left(e^{v}\right)}{d v^{\lambda}} \equiv \frac{1}{\lambda} \frac{d_{0}^{\lambda} l f\left(e^{r v}\right)}{d v^{\lambda}} \equiv r^{\lambda} \cdot \frac{1}{\lambda} \frac{d_{0}^{\lambda} l f\left(e^{\nu}\right)}{d v^{\lambda}}, \quad(\bmod . \lambda),
$$

also, weil $r^{\lambda} \equiv r$, nicht $\equiv 1$ ist,

$$
\frac{1}{\lambda} \frac{d_{0}^{\lambda} l f\left(e^{v}\right)}{d v^{\lambda}} \equiv 0, \quad(\bmod . \lambda) \text {. }
$$

Ferner hat man, weil $f(\alpha)=f\left(\alpha^{r}\right)$ ist,

$$
\left(\frac{\lambda}{f(\alpha)}\right)=\alpha^{\text {Ind. } \lambda,}, \quad\left(\frac{\lambda}{f^{\prime}\left(\alpha^{r}\right)}\right)=\alpha^{\text {Ind. } \lambda,}
$$

und wenn man in der ersten dieser beiden Gleichungen $\alpha^{r}$ statt $\alpha$ setzt

$$
\left(\frac{\lambda}{f\left(\alpha^{r}\right)}\right)=\alpha^{r \text { Ind. } \lambda}
$$

und demnach

$$
\alpha^{r \text { Ind. } \lambda}=\alpha^{\text {Ind. } \lambda,} \quad r \text { Ind. } \lambda \equiv \operatorname{Ind} . \lambda, \quad(\bmod . \lambda),
$$

woraus, weil $r$ nicht congruent Eins ist, Ind. $\lambda \equiv 0$ folgt.

\section{3.}

Aus dem gefundenen Ausdrucke (11.) des Index von $\lambda$, dessen Allgemeingültigkeit für alle complexen Primzahlen $f(\alpha)$ hierdurch bewiesen ist, soll nun mit Hülfe der in der angeführten Abhandlung entwickelten Indices der Einheiten ein neuer fur alle Primzahlen $f(\alpha)$ geltender Ausdruck des Index vòn $1-\alpha^{x}$ hergeleitet werden; in ähnlicher Weise, wie dies a. a. 0. pag. 129 für den speciellen Fall ausgeführt ist, wo Ind. $\lambda \equiv 0,(\bmod . \lambda)$ ist.

Aus der Formel

$$
\lambda=(1-\alpha)\left(1-\alpha^{\gamma}\right)\left(1-\alpha^{\gamma^{2}}\right) \ldots\left(1-\alpha^{\gamma^{\lambda-2}}\right),
$$

in welcher $\gamma$ eine primitive Wurzel von $\lambda$ bezeichnet, erhält man

$$
\lambda=(1-\alpha)^{\lambda-1} \alpha^{-\mu} e(\alpha)^{\lambda-1} e\left(\alpha^{\gamma}\right)^{\lambda-2} e\left(\alpha^{\gamma^{2}}\right)^{\lambda-3} \ldots e\left(\alpha^{\gamma^{\lambda-2}}\right)^{l},
$$

wo $\mu=\frac{\lambda-1}{2}$, und wo $e(\alpha)$ die Kreistheilungseinheit

$$
e(\alpha)=\sqrt{\frac{\left(1-\alpha^{\gamma}\right)\left(1-\alpha^{-\gamma}\right)}{(1-\alpha)\left(1-\alpha^{-1}\right)}}
$$

ist. Verwandelt man $\alpha$ in $\alpha^{\alpha}$ und nimmt auf beiden Seiten die Indices nach 
dem Modul $f(\alpha)$, so erhält man

$$
\text { (13.) Ind. } \left.\lambda \equiv-\text { Ind. }\left(1-\alpha^{\nu}\right)-\mu \varkappa \operatorname{Ind} . \alpha-\sum_{0}^{x-1} h \operatorname{Ind} . e\left(\alpha^{\alpha \gamma^{h-1}}\right) \text {, (mod. } \lambda\right) \text {. }
$$

Drückt man nun die einfachen Kreistheilungseinheiten $e(\alpha), e\left(\alpha^{\gamma}\right), \ldots$ durch die zusammengesetzten Kreistheilungseinheiten $\mathbf{E}_{1}(\alpha), \mathbf{E}_{2}(\alpha), \ldots \mathbf{E}_{\mu-1}(\alpha)$ aus, welche durch die Gleichung

$$
\mathbf{E}_{n}(\alpha)=\boldsymbol{e}(\alpha) \boldsymbol{e}\left(\alpha^{\gamma}\right)^{\gamma^{-2 n}} \boldsymbol{e}\left(\alpha^{\gamma^{2}}\right)^{\gamma^{-4 n}} \ldots e\left(\alpha^{\gamma^{\mu-1}}\right)^{\gamma^{-2(\mu-1) n}}
$$

bestimmt sind, aus welcher

$$
\text { Ind. } E_{n}(\alpha) \equiv \sum_{0}^{\mu-1} \gamma^{-2 n h} \operatorname{Ind} . e\left(\alpha^{\gamma^{h}}\right), \quad(\bmod . \lambda)
$$

und durch Umkehrung

$$
\text { Ind. } e\left(\alpha^{\gamma^{h}}\right) \equiv-2 \sum_{1}^{\mu-1} \gamma^{2 n h} \operatorname{Ind} . \mathbf{E}_{n}(\alpha),(\bmod . \lambda)
$$

folgt, so erhält man aus der Congruenz (13.)

(14.) Ind. $\lambda \equiv-$ Ind. $\left(1-\alpha^{\varkappa}\right)-\mu \varkappa \operatorname{Ind} . \alpha+2 \underset{1}{\lambda-1} \sum_{1}^{\mu-1} \sum_{n} h \gamma^{2 n(h-1)} \operatorname{Ind} . \mathrm{E}_{n}\left(\alpha^{\alpha}\right)$.

Führt man die Summation in Beziehung auf $h$ aus und bringt Ind. $\left(1-\alpha^{x}\right)$ allein auf eine Seite der Congruenz, so hat man

$$
\text { (15.) Ind. }\left(1-\alpha^{\alpha}\right) \equiv-\text { Ind. } \lambda-\mu \varkappa \operatorname{Ind} . \alpha-2 \sum_{1}^{\mu-1} \frac{\operatorname{Ind} . E_{n}\left(\alpha^{\alpha}\right)}{\gamma^{2 n}-1},(\bmod . \lambda) \text {. }
$$

Macht man nun von dem a. a. 0. pag. 128 gegebenen Index der Einheit $\mathbf{E}_{n}(\alpha)$ Gebrauch:

(16.) Ind. $\mathbf{E}_{n}\left(\alpha^{\alpha}\right) \equiv(-1)^{n}\left(\gamma^{2 n}-1\right) \frac{B_{n} x^{\lambda-2 n}}{4 n} \frac{d_{0}^{\lambda-2 n} l f\left(e^{v}\right)}{d v^{\lambda-2 n}}, \quad(\bmod . \lambda)$, in welchem $\boldsymbol{B}_{n}$ die $\boldsymbol{n}^{\text {te }}$ Bernoullische Zahl ist, und setzt der Kürze halber

$$
\frac{d_{0}^{x} l f\left(e^{v}\right)}{d v^{x}}=D_{x} \text {, }
$$

so erhält man, indem man für Ind. $\lambda$ den gefundenen Werth bei (11.) und für Ind.( $\alpha)$ seinen Werth $\frac{N f(\alpha)-1}{\lambda}$ setzt, folgenden Ausdruck des Index von $1-\alpha^{x}$ :

$$
\begin{gathered}
\text { (17.) Ind. }\left(1-\alpha^{x}\right) \equiv \\
-\frac{D_{\lambda}}{\lambda}+\frac{1}{2} \frac{N f(\alpha)-1}{\lambda} \varkappa+B_{1} D_{\lambda-2} \frac{x^{2}}{2}-B_{2} D_{\lambda-4} \frac{x^{4}}{4}+\cdots+(-1)^{\mu} B_{\mu-1} D_{3} \frac{x^{\lambda-3}}{\lambda-3},
\end{gathered}
$$

nach dem Modul $\lambda$. 


\section{4.}

Aus den Indices der besonderen Einheiten $\mathbf{E}_{1}(\alpha), \mathbf{E}_{2}(\alpha), \ldots \mathbf{E}_{\mu-1}(\alpha)$ läfst sich ein allgemeiner Ausdruck für den Index einer jeden beliebigen Einheit $E(\alpha)$ auf folgende Weise ableiten. Da das System dieser $\mu-1$ Einheiten ein vollständiges unabhängiges System ist, so läfst sich jede gegebene Einheit $\boldsymbol{E}(\alpha)$ durch dasselbe ausdrücken in der Form

$$
\text { (18.) } \quad E(\alpha)=\alpha^{m} E_{1}(\alpha)^{m_{1}} E_{2}(\alpha)^{m_{2}} \ldots E_{\mu-1}(\alpha)^{m_{\mu-1}},
$$

aus welcher man

(19.) Ind. $E(\alpha)=m$ Ind. $\alpha+m_{1}$ Ind. $E_{1}(\alpha)+m_{2}$ Ind. $E_{2}(\alpha)+\cdots+m_{\mu-1}$ Ind. $E_{\mu-1}(\alpha)$ erhält, wo $m_{1}, m_{2}, \ldots m_{\mu-1}$ auch rationale Brüche sein können, aber nicht solche, welche in ihren Nennern $\lambda$ enthalten, wenn nämlich, wie vorausgesetzt worden ist, $\lambda$ nicht in einer der ersten $\frac{\lambda-3}{2}$ Bernoullischen Zahlen als Factor des Zählers enthalten ist. Verwandelt man nun in der Form (18.) die Wurzel $\alpha$ in die Variable $e^{v}$ und differentiirt logarithmisch, so werden alle ungraden Differentialquotienten des Logarithmus von $\mathbf{E}_{n}\left(\boldsymbol{e}^{v}\right)$ congruent Null, nach dem Modul $\lambda$, wegen der Eigenschaft dieser Einheit, nach welcher $\mathbf{E}_{n}(\alpha)=\mathbf{E}_{n}\left(\alpha^{-1}\right)$ ist. Ferner hat man

$$
\frac{d_{0}^{2 x} l \mathrm{E}_{n}\left(e^{v}\right)}{d v^{2 x}} \equiv \sum_{0}^{\mu-1} \gamma^{-2 n h} \frac{d_{0}^{2 x} l e\left(e^{v \gamma^{h}}\right)}{d v^{2 x}} \equiv \frac{d_{0}^{2 x} l e\left(e^{v}\right)}{d v^{2 x}} \sum_{0}^{\mu-1} \gamma_{h}^{(2 x-2 n) h},(\bmod . \lambda),
$$

und weil diese Summe stets congruent Null ist, mit Ausnahme des Falles $\varkappa=n$, in welchem sie congruent $\mu$ wird, nach dem Modul $\lambda$, so hat man

und weil

$$
\text { (20.) }\left\{\begin{array}{l}
\frac{d_{0}^{2 *} l \mathrm{E}_{n}\left(e^{v}\right)}{d v^{2 \varkappa}} \equiv 0,(\bmod . \lambda), \text { wenn } * \text { nicht gleich } n, \\
\frac{d_{0}^{2 n} l \mathrm{E}_{n}\left(e^{v}\right)}{d v^{2 n}} \equiv \mu \frac{d_{0}^{2 n} l e\left(e^{v}\right)}{d v^{2 n}},(\bmod . \lambda),
\end{array}\right.
$$

$$
\frac{d_{0}^{2 n} l e\left(e^{v}\right)}{d v^{2 n}} \equiv(-1)^{n+1}\left(\gamma^{2 n}-1\right) \frac{\boldsymbol{B}_{n}}{2 n},(\bmod . \lambda),
$$

wie in der angeführten Abhandlung pag. 139 gezeigt worden ist, so ist

$$
\text { (21.) } \frac{d_{0}^{2 n} l \mathrm{E}_{n}\left(e^{v}\right)}{d v^{2 n}} \equiv(-1)^{n}\left(\gamma^{2 n}-1\right) \frac{\boldsymbol{B}_{n}}{4 n},(\bmod . \lambda) \text {. }
$$

Man erhält demnach aus der Gleichung (18.) folgenden Werth des $2 n^{\text {ten }}$ Differentialquotienten von $l \boldsymbol{E}\left(\boldsymbol{e}^{v}\right)$ :

$$
\frac{d_{0}^{2 n} l E\left(e^{v}\right)}{d v^{2 n}} \equiv m_{n} \frac{d_{0}^{2 n} l \mathrm{E}_{n}\left(e^{v}\right)}{d v^{2 n}} \equiv m_{n}(-1)^{n}\left(\gamma^{2 n}-1\right) \frac{\boldsymbol{B}_{n}}{4 n},
$$


und wenn mit $\frac{d_{0}^{\lambda-2 n} l f\left(e^{v}\right)}{d v^{\lambda-2 n}}$ multiplicirt wird:

$$
\frac{d_{0}^{2 n} l E\left(e^{v}\right)}{d v^{2 n}} \frac{d_{0}^{\lambda-2 n} l f\left(e^{v}\right)}{d v^{\lambda-2 n}} \equiv m_{n}(-1)^{n}\left(\gamma^{2 n}-1\right) \frac{B_{n}}{4 n} \frac{d_{0}^{\lambda-2 n} l f\left(e^{v}\right)}{d v^{\lambda-2 n}}
$$

also vermöge der Formel (16.)

$$
\frac{d_{0}^{2 n} l E\left(e^{v}\right)}{d v^{2 n}} \frac{d_{0}^{\lambda-2 n} l f\left(e^{v}\right)}{d v^{\lambda-2 n}} \equiv m_{n} \text { Ind. } \mathbf{E}_{n}(\alpha)
$$

Der Ausdruck des Index der beliebigen complexen Einheit $\boldsymbol{E}(\alpha)$ wird daher, weil überdies $\frac{d_{0} l E\left(e^{v}\right)}{d v} \equiv m$ ist:

(22.) Ind. $E(\alpha) \equiv \frac{d_{0} l E\left(e^{v}\right)}{d v} \frac{N f(\alpha)-1}{\lambda}+\sum_{1}^{\mu-1} \frac{d_{0}^{2 n} l E\left(e^{v}\right)}{d v^{2 n}} \frac{d_{0}^{\lambda-2 n} l f\left(e^{v}\right)}{d v^{\lambda-2 n}},(\bmod . \lambda)$. Berlin, im December 1858. 